\title{
Analysis of the Shear Viscosity in Quaternary Mixtures of Rarefied Gases
}

\author{
Adriano Willian da Silva \\ Kayk Bueno Martins \\ Rafael Lima Princival
}

Instituto Federal do Parana, Campus Curitiba, PR, Brazil

adriano.silva@ifpr.edu.br, kaykbuenomartins@gmail.com, rafaprincival99@ hotmail.com

\begin{abstract}
In this work, we consider a dilute reactive mixture of four constituents undergoing the reversible reaction $A+B \rightleftharpoons C+D$. The mixture is described using the Boltzmann equation (BE) to chemically reactive systems which treats both elastic and reactive collisions as hard spheres type. We use the Chapman-Enskog method, at the first-order level of the Enskog expansion, to determine the non-equilibrium solution to the system in a chemical regime for which both elastic and reactive collisions occur with comparable characteristic times. In this case, the chemical reaction can be considered in its final stage, close to chemical equilibrium (fast process). We then determine the transport coefficients and focus our analysis on the evaluation of the coefficient associated with the shear viscosity and investigate how the reaction heat and the activation energy changes this coefficient.
\end{abstract}

\section{Introduction}

Non-equilibrium effects and transport properties of chemically reactive mixtures are widely investigated in modern fluid dynamics, due to several applications of multicomponent reactive systems in chemical engineering, biotechnology, combustion engineering, propulsion devices and other industrial processes. The description of the chemical kinetics of the reaction mechanism is an important part of the investigation and the application of mathematical models can provide some additional tools to better under- stand the chemical process [1]. Several papers appear in the literature concerning the study of the Boltzmann equation applied to chemical processes and among others we quote the works of Present [3], Ross and Mazur [4], Shizgal and Karplus [5], Cukrowski and Popielawski [7].

The present paper also arises in this direction. In the kinetic model here used, elastic and reactive collisions are assumed of hard-sphere type and are treated of the same order. Therefore, this model is appropriate to investigate processes in which chemical reactions play a relevant 


\section{Blucher Proceedings IX Encontro Científico de Física Aplicada Blucher}

role. This work is applied to a quaternary reactive mixture of monatomic gases, whose constituents undergo the reversible reaction of bimolecular type. We use the Chapman-Enskog method and the second approximation of the Enskog expansion, to determine the non-equilibrium solution to the system in a chemical regime of fast chemical reaction for which both elastic and reactive collisions occur with comparable characteristic times.

In this paper, we start with a simpler case in the dilute gas regime and focus our analysis on the evaluation of the transport coefficients associated to shear viscosity of the reactive mixture.

\section{Boltzmann Equation}

We consider a quaternary mixture of ideal gases whose constituents denoted by $A$, $B, C$ and $D$, have binding energies $\varepsilon_{\alpha}=$ $A, B, C, D$. There exist two kinds of collisions between the molecules of the gas: the elastic one which refers to non reactive interactions and the one which takes into account the reaction. The gas molecules undergo inelastic collisions with reversible reactions of the type

$$
A+B \rightleftharpoons C+D
$$

The conservation laws of linear momentum and total energy for a reactive collision are given by

$$
\begin{gathered}
m_{A} \mathbf{c}_{\mathbf{A}}+m_{B} \mathbf{c}_{\mathbf{B}}=m_{C} \mathbf{c}_{\mathbf{C}}+m_{D} \mathbf{c}_{\mathbf{D}}, \\
\varepsilon_{A}+\frac{1}{2} m_{A} c_{A}^{2}+\varepsilon_{B}+\frac{1}{2} m_{B} c_{B}^{2} \\
=\varepsilon_{C}+\frac{1}{2} m_{C} c_{C}^{2}+\varepsilon_{D}+\frac{1}{2} m_{D} c_{D}^{2},
\end{gathered}
$$

where $\left(\mathbf{c}_{\mathbf{A}}, \mathbf{c}_{\mathbf{B}}\right)$ and $\left(\mathbf{c}_{\mathbf{C}}, \mathbf{c}_{\mathbf{D}}\right)$ are the velocities of reactants and products, respectively, of the forward reaction. The conservation laws for the nonreactive collisions have the same form as the above equations.

We denote by $\mathbf{g}_{\mathbf{B A}}=\mathbf{c}_{\mathbf{B}}-\mathbf{c}_{\mathbf{A}}$ and $\mathbf{g}_{\mathbf{D C}}=\mathbf{c}_{\mathbf{D}}-\mathbf{c}_{\mathbf{C}}$ the assymptotic relative velocities of the reactants and products of the forward reaction, respectively, and write the conservation law of total energy (3) as

$$
\begin{gathered}
\frac{1}{2} m_{A B} g_{B A}^{2}=\frac{1}{2} m_{C D} g_{C D}^{2}+E, \\
E=\varepsilon_{D}+\varepsilon_{C}-\varepsilon_{B}-\varepsilon_{A}=\varepsilon-\varepsilon_{r},
\end{gathered}
$$

where $E$, the difference between the binding energies of products and reactants is connected with the reaction heat.

In the phase space defined by positions and velocities of particles, the state of the mixture is defined in terms of the set of one-particle distribution functions

$$
f_{\alpha}=f\left(\mathbf{x}, \mathbf{c}_{\alpha}, t\right) \quad(\alpha=A, B, C, D)
$$

such that $f_{\alpha} d \mathbf{x} d \mathbf{c}_{\alpha}$ gives at time $t$ the number of molecules of type $\alpha$ in the volume element $d \mathbf{x} d \mathbf{c}_{\alpha}$ around the position $\mathbf{x}$ and the velocity $\mathbf{c}_{\alpha}$. The evolution of te one-particle distribution $f_{\alpha}$ in the phase space is assumed to satisfy the Boltzmann equation which in the absence of external forces is written as

$\frac{\partial f_{\alpha}}{\partial t}+c_{i}^{\alpha} \frac{\partial f_{\alpha}}{\partial x_{i}}=Q_{\alpha}^{E}+Q_{\alpha}^{R}, \quad(\alpha=A, B, C, D)$

where

$$
Q_{\alpha}^{E}=\sum_{\beta=A}^{D} \int\left[f_{\alpha}^{\prime} f_{\beta}^{\prime}-f_{\alpha} f_{\beta}\right] d \Gamma_{\alpha \beta}
$$


$Q_{A B}^{R}=\int\left[f_{C} f_{D}\left(\frac{m_{A B}}{m_{C D}}\right)^{3}-f_{A} f_{B}\right] d \Gamma_{A B}^{\star}$,

$Q_{C D}^{R}=\int\left[f_{A} f_{B}\left(\frac{m_{C D}}{m_{A B}}\right)^{3}-f_{C} f_{D}\right] d \Gamma_{C D}^{\star}$

and

$$
\begin{gathered}
d \Gamma_{\alpha \beta}=d^{2}\left(\mathbf{g}_{\beta \alpha} \cdot \mathbf{k}_{\beta \alpha}\right) d \mathbf{k}_{\beta \alpha} d \mathbf{c}_{\beta} \\
d \Gamma_{A B}^{\star}=\sigma_{A B}^{\star}\left(\mathbf{g}_{B A} \cdot \mathbf{k}_{B A}\right) d \mathbf{k}_{B A} d \mathbf{c}_{B}, \\
d \Gamma_{C D}^{\star}=\sigma_{C D}^{\star}\left(\mathbf{g}_{D C} \cdot \mathbf{k}_{D C}\right) d \mathbf{k}_{D C} d \mathbf{c}_{D}
\end{gathered}
$$

The quantities $\sigma_{B A}^{\star}$ and $\sigma_{D C}^{\star}$ denote reactive differential cross section, $\mathbf{k}_{\alpha}$ and $d \mathbf{k}_{\alpha}$ denote the unit collision vector and the element of solid angle for elastic collisions, whereas $\mathbf{k}_{\alpha}$ and $d \mathbf{k}_{\alpha}$ represent the corresponding quantities or reactive interactions. The parameter $d$ denotes the diameter of a particle. Due to the principle of microscopic reversibility [10], a definite relationship exists between the forward cross section $\sigma_{B A}^{\star}$ and that for the reverse reaction $\sigma_{D C}^{\star}$. The parameter $d$ denotes the diameter of a particle. Due to the principle of microscopic reversibility [10], there is relationship exists between the forward cross section $\sigma_{A B}^{\star}$ and that for the reverse reaction $\sigma_{C D}^{\star}$. By using this principle, the transformation law bettween the elements for the reactive and forward collisions in the velocity space is given by

$$
\begin{gathered}
m_{C D} g_{B A} d \mathbf{k}_{B A} d \mathbf{c}_{C} d \mathbf{c}_{D} \\
=m_{A B} g_{D C} d \mathbf{k}_{D C} d \mathbf{c}_{A} d \mathbf{c}_{B}, \\
m_{C D}^{2} g_{D C}^{2} \sigma_{C D}^{\star}=m_{A B}^{2} g_{B A}^{2} \sigma_{A B}^{\star} .
\end{gathered}
$$

$f_{\alpha}, \alpha=A, B, C, D$ and it is characterized after solving the appropriate reactive Boltzmann equation (6). This can be achieved in the framework of the Chapman-Enskog method [2, 3], once line-of-center model for the reactive cross sections is assumed,

$$
\begin{gathered}
\sigma_{A B}^{\star}=0, \quad \varepsilon_{\alpha}<\varepsilon^{\star} \\
\sigma_{A B}^{\star}=d r^{2}\left(1-\frac{\varepsilon^{\star}}{\gamma_{A B}}\right), \quad \gamma_{A B}>\varepsilon^{\star}
\end{gathered}
$$

In equation (12) $\gamma_{A B}=m g_{B A}^{2} / 4 k T$ is the relative translational energy of constituent $\alpha$ and $\varepsilon^{\star}$ is the activation energies of the reaction in units of the thermal energy of the mixture, $k T, T$ is the temperature of the mixture and $k$ being the Boltzmann constant.

If we multiply the Boltzmann equation (6) by arbitrary function $\psi_{\alpha}=$ $1, \psi_{\alpha}=m c_{i}^{\alpha}$ and $\psi_{\alpha}=m c_{\alpha}^{2} / 2+\varepsilon_{\alpha}$, respectively and integrate the resulting equations over all values of $\mathbf{c}_{\alpha}$, we get the equations

$$
\begin{aligned}
& \frac{\partial n_{\alpha}}{\partial t}+\frac{\partial}{\partial t}\left(n_{\alpha} v_{i}+n_{\alpha} u_{i}^{\alpha}\right) \\
& =\int\left(Q_{\alpha}^{E}+Q_{\alpha}^{R}\right) d \mathbf{c}_{\alpha}=\tau_{\alpha},
\end{aligned}
$$

$$
\frac{\partial}{\partial t}\left(m_{\alpha} n_{\alpha}\right) v_{i}^{\alpha}+\frac{\partial}{\partial x_{j}}\left[p_{i j}^{\alpha}+m_{\alpha} n_{\alpha}\left(u_{i}^{\alpha} v_{j}\right.\right.
$$$$
\left.\left.+u_{j}^{\alpha} v_{i}+v_{i} v_{j}\right)\right]=\int m_{\alpha} c_{i}^{\alpha}\left(Q_{\alpha}^{E}+Q_{\alpha}^{R}\right) d \mathbf{c}_{\alpha},
$$

$$
\frac{\partial}{\partial t}\left[\frac{3}{2} n_{\alpha} k T+n_{\alpha} \varepsilon_{\alpha}+m_{\alpha} n_{\alpha}\left(u_{i}^{\alpha} v_{i}+\frac{1}{2} v^{2}\right)\right]
$$

\section{Chapman-Enskog Method $+\frac{\partial}{\partial x_{i}}\left\{q_{i}^{\alpha}+p_{i j}^{\alpha} v_{j}+n_{\alpha} \varepsilon_{\alpha} u_{i}^{\alpha}+\frac{1}{2} m_{\alpha} n_{\alpha} v^{2} u_{i}^{\alpha}\right.$ \\ The non-equilibrium effect are contained in the one-particle distribution functions,

$$
+\left[\frac{3}{2} n_{\alpha} k T+n_{\alpha} \varepsilon_{\alpha}+m_{\alpha} n_{\alpha}\left(u_{i}^{\alpha} v_{i}+\frac{1}{2} v^{2}\right)\right] v_{i}
$$




\section{Blucher Proceedings \\ IX Encontro Científico de Física Aplicada Blucher}

$$
=\int\left(\frac{m_{\alpha}}{2} c_{\alpha}^{2}\right)\left(Q_{\alpha}^{E}+Q_{\alpha}^{R}\right) d \mathbf{c}_{\alpha},
$$

where $n_{\alpha}$ is particle densities, $v_{i}^{\alpha}$ is the velocity components, $T$ is the temperature of the mixture, $u_{i}^{\alpha}$ is the diffusion velocity, $p_{i j}^{\alpha}$ is pressure tensor, $q_{i}^{\alpha}$ is the heat flux for each constituent $\alpha$ and $\tau_{\alpha}$ denotes the rate of reaction due to the chemical reaction. The quantities above are defined in terms of the distribution function [6].

The distribution function contains all the information about the nonequilibrium effects induced by the chemical reaction. The solution of the Boltzmann equation (6) in a chemical regime for which the reaction process is close to its final stage (fast process) to this problem is based on the Chapman-Enskog method and Sonine polynomial approximation to the coefficients of the distribution functions [11].

For the solution of the Boltzmann equation (6) it is convenient to write $f_{\alpha}$ in terms of Sonine polynomials and retain, at least, the expansion up to the first-order term,

$$
\begin{aligned}
& f_{\alpha}=f_{\alpha}^{M}\left\{1+b_{0}^{\alpha} \xi_{<i}^{\alpha} \xi_{j>}^{\alpha} \frac{m_{\alpha}}{k T} \frac{\partial v_{<i}}{\partial x_{j>}}\right\}, \\
& f_{\alpha}^{M}=n_{\alpha}\left(\frac{m_{\alpha}}{2 \pi k T}\right)^{3 / 2} \exp \left(-\frac{m_{\alpha} \xi_{\alpha}^{2}}{2 k T}\right)
\end{aligned}
$$

where $b_{0}^{\alpha}$ is scalar coefficient to be determined The expansion adopted is capable to reproduce an appreciate effect of the reaction heat and reactive cross section on the distribution function.

\section{Results}

The pressure tensor of the mixture is defined by

$$
\begin{gathered}
p_{i j}=\sum_{\alpha=A}^{D} \int m_{\alpha} \xi_{i}^{\alpha} \xi_{j}^{\alpha} f_{\alpha} d \mathbf{c}_{A}= \\
p \delta_{i j}-2 \mu \frac{\partial v_{<i}}{\partial x_{j>} .}
\end{gathered}
$$

Where $\mu$ is the shear viscosity of the mixture.

The figures shown the dimensionless shear viscosity with $m_{A}=2,2 ; m_{B}=1,8$; $m_{C}=3,8$ and $m_{D}=0,2$.

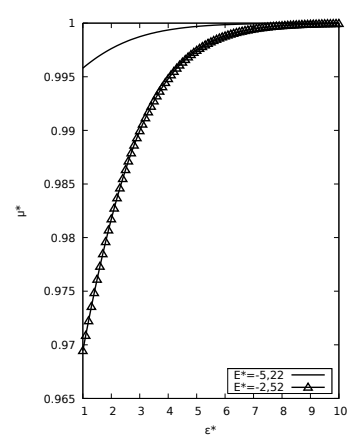

Figure 1: Dimensionless Shear Viscosity Coefficient as function of the activation energy $\varepsilon^{\star}$ to exothermics reactions

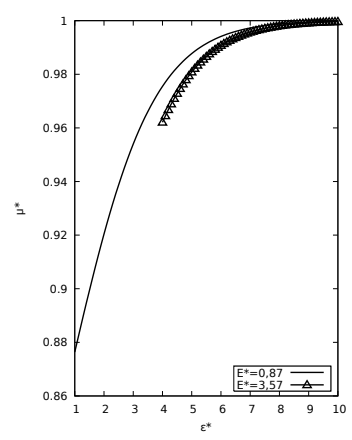

Figure 1: Dimensionless Shear Viscosity Coefficient as function of the activation energy $\varepsilon^{\star}$ to endothermics reactions

In figures 1 and 2 are plotted respectively, the dimensionless shear viscosity coefficient $\mu^{\star}=\mu / \mu^{I}$ to exothermics and endothermics reactions and $\mu^{I}$ is shear viscosity for inert mixture.

With respect to the viscosity coefficient the figures 1 and 2 have shown that the non-equilibrium effects induced by the chemical reaction are more noticeable for small values of the activation energy, 


\section{Blucher Proceedings IX Encontro Científico de Física Aplicada Blucher}

as expected. The effects are more noticeable when the endothermic reaction is predominant and, in addition, become greater when the endothermicity (respectively exothermicity) character increases (respectively decreases) Besides, the viscosity of the reactive mixture is smaller than the one of the inert mixture.

\section{Conclusions}

In this paper, the second approximation to the distribution functions were determined from the system of Boltzmann equations for the last stage of a chemical reaction- known as fast reaction- where the affinity is considered as a small quantity in comparison with the thermal energy of the mixture. The reaction heat modifies the shear viscosity, i. e., the production term $p_{i j}$ of the pressure tensor of the mixture and the effect is more acenttuated to low activation energies. It is shown the reaction to occur only when the relative translational energy is greater than the activation energy.

\section{References}

[1] R. D. Levine, Molecular Reaction Dynamics, Cambridge University Press, 2005.

[2] I. Prigogine and E. Xhrouet, On the perturbation of Maxwell distribution function by chemical reaction in gases, Physica, Amsterdam,15: 913-932, 1949.

[3] R. D. Present, Note on the simple collision theory of bimolecular reactions, Proc. Natl. Acad. Sci. U.S.A. 41: 415-417, 1995.
[4] J. Ross and P. Mazur, Some deductions from a formal statistical mechanical theory of chemical kinetics, J. Chem. Phys. 35: 19-28, 1960.

[5] B. D. Shizgal and M. Karplus, Nonequilibrium contributions to the rate of reactions I. Perturbation of the velocity distribution function, $\mathrm{J}$. Chem. Phys. 52: 4262-4278, 1970.

[6] A. W. Silva, G. M. Alves and G. M. Kremer, Transport phenomena in a reactive quaternary gas mixture, Physica A 374: 533-548, 2007.

[7] A. S. Cukrowski, J. Popielawski, L. Qin and J. S. Dahler, A simplified theoretical analysis of nonequilibrium effects in bimolecular gas phase reactions, J. Chem. Phys., 97: 9086-9093, 1992.

[8] W. Stiller, Arrhenius equation and a non equilibrium kinetics, Teubner, Leipzig, 1990

[9] G. M. Kremer, An introduction to the Boltzmann equation and transport process in gases. Springer, Berlin, 2010.

[10] J. C. Light, J. Ross and K. E. Shuler, In Kinetic Processes in Gases and Plasmas, edited by A. R. Hochstim. Academic, New York, p. 281-321, 1969.

[11] B. Shizgal and A. Chikhaoui, On the use temperature parameterized rate coefficients in the estimation of nonequilibrium reaction rates, Physica A 365: 317-332, 1996. 